\title{
Improving Magnetic Coupling for Battery Charging Through 3D Magnetic Flux
}

\author{
Luis F. Romba Jorge, Stanimir S. Valtchev \\ UNINOVA-CTS and Faculdade de Ciências e \\ Tecnologia, Universidade Nova de Lisboa, Portugal \\ luis.rjorge@netvisao.pt; ssv@fct.uni.pt
}

\author{
Rui Melício \\ IDMEC/LAETA, Instituto Superior Técnico \\ Universidade de Lisboa, Departamento de Física, \\ Escola de Ciências e Tecnologia, Universidade de \\ Évora, Portugal,ruimelicio@gmail.com
}

\begin{abstract}
The spatial distribution of the magnetic field and the coupling between the coils in the Wireless Power Transfer (WPT) systems is an important aspect to consider in the system design and efficiency optimization. The presented study in this paper is based on tests performed on a physical model. The transmitting (primary) equipment, is an electrical three-phase system, capable to be connected in star or delta (both electrically and geometrically). The measured results allow to describe graphically the magnetic field distribution in three dimensions. The analytical formulas aim to help to understand and to quantify the physical phenomena but they cannot be considered a universal approach and the measurement results help to understand better the observable facts. In the WPT, the key issues that will influence the efficiency, are the alignment of the coils, the spatial orientation of the magnetic field, the detachment and the tilt between the windings, all they changing the magnetic coupling between the transmitter and the receiver of energy. This research is directed not only to the magnetic field distribution but finally, to optimize the energy transfer efficiency.
\end{abstract}

Keywords - WPT, Magnetic field, Magnetic Resonance Circuits (MRC), IPT, Three-Phase system

\section{INTRODUCTION}

The concept of the electric power transmission, over certain distances without any carrier medium (i.e. wirelessly), had its beginning with the work developed by Nikola Tesla [1]. For long time the technology associated to these works was forgotten and only recently, with the advent of the portable entertainment and communication devices appeared the opportunity to look again at this technology [2]. The development of the WPT technologies, has shown that it is possible to be used in many potential applications. The applications can be industrial, medical and wireless charging for hybrid and electrical vehicles [3]. The nature of the noncontact power transmission system, allows to have many advantages: to be not affected by the environmental conditions, because electronic components can be completely sealed; to be robust in operation and inherently safe, because of the isolated system, since there is no need to handle any electrical cables [4] [7]. The applications of the WPT systems have been growing in number: clean-room systems, material handling monorail-based systems (often, automotive assembly) when the environment is extremely dirty, slow-moving automatic guided vehicles (AGVs) with track-following navigation, and the battery-charging systems. Other applications are in the biomedicine, the aerospace systems, the electric vehicles and the robotics [7-8]. There are three conditions that all wireless power systems must satisfy to work as battery charger: to obtain high efficiency, to support large air gaps and to transmit a high power [4].

The WPT can be categorized in two types, radiative or nonradiative, depending on the process used to transfer the energy. In WPT of radiative type, the energy is emitted from an antenna and propagates through the vacuum or air [5]. This type of power transmission using directed microwaves beams for connecting the transmitter to the receiver, requires accurate alignment and clear visual contact [6]. The non-radiative energy transfer [1] connects directly the transmitter and the receiver by magnetic (rarely by electric) field. Based on the distance of the energy transfer, the non-radiative (magnetic, electric or electromagnetic field) coupling can be categorized into a short-range (Electromagnetic Induction) and a midrange (Strongly Coupled Magnetic Resonance, abbreviated as SCMR). Concerning the electromagnetic induction (the usual WPT), the transfer efficiency and the transferred power are quite high, but the transfer distance is limited to centimeters. On the contrary, in the SCMR the transfer efficiency and the transferred power is slightly lower, but the transfer distance can reach a few meters [11] The non-radiative contactless energy transfer, is generally based on the near field magnetic coupling between two or more coils. This process can be aimed for use in short and medium range applications [5]. In the electromagnetic resonance coupling, the physical dimensions of the transmitter coil should never be equal or close to $\lambda / 2$, where $\lambda$ is the wavelength of the transmitted power. When these conditions are considered, the system is not radiative, existing only magnetic field [10], or rarely, electric field. For many reasons, it is easier to transfer the energy by magnetic induction. In the inductive power transfer (IPT) systems, one coil transfers energy to another due to the mutual inductance between these two coils. The basic rules that manage this phenomenon are similar to the transformers, but while in the transformer the magnetic coupling is strong on both sides, primary and secondary, the IPT systems coupling is weak, due to the large air gap between the coils [9]. With the continuous evolution of the research related to the WPT systems, there have been more and more applications. mainly in the area of electric mobility [11]. Nowadays, due to the announced depletion of the fossil fuel reserves and the successive geopolitical crises in the areas of higher extraction and 
production of such fuels, there has been an increased demand for alternative / renewable energy, which can easily be converted into electrical power. In the same time the internal combustion (IC) vehicles are substituted rapidly by electric vehicles (EV). The EVs in turn, need energy and it is considered most convenient to use a contactless transfer.

It is already a common practice the connection of the hybrid vehicle or electric vehicle to the grid to charge their batteries, i.e. the connection is grid-to-vehicle (G2V). However, it has not been accepted easily the concept of vehicle-to-grid (V2G), which uses hybrid vehicles or pure electric vehicle to store and supply energy back to the grid [12]. The resonant IPT method could be a good candidate for the bidirectional energy transfer.

To build up a resonant circuit two methods can be used: LC Series (S) or LC Parallel (P), giving four basic circuits: SS, SP, PS and PP, (first letter denotes primary circuit and second letter denotes the secondary circuit) [14]. The coupling factor $k$ is a very important issue for the magnetic coupled resonance WPT system. It represents how much power from the generated electromagnetic field (system primary) is induced in the secondary coil. The coupling factor is determined by the distance between the coils, their size, shape and the angle between them [13] [15].

\section{MODELING}

When an electric current flows through a conductor, a magnetic field is generated. The constant current will induce a constant magnetic field. However, a time-varying current will generate a varying magnetic flux. If a second conductor is placed nearby, an induced emf will be created in each turn, as stated by Faraday's Law and given by the following equation:

$$
\mathrm{E}=-\frac{\partial \Phi_{B}}{\partial t}
$$
force.

Here, $\Phi_{B}$ is the magnetic flux and $E$ is the electromotive

For a generic closed path around a conductor with the incremental length $d l$, with the same direction of magnetic field, the magnetic flux density $B$ is a function of the current through the conductor given by:

$$
\int B . d l=i . \mu_{0}
$$

Here $\mu_{0}$ is the vacuum permeability. The path of $B$ around the straight conductor is always circular, so at a distance $r$ from the center of the conductor, the integral of the (2) is reduced to:

$$
\int B . d l=B .2 \pi r
$$

Since the magnetic field intensity is inversely proportional to the distance from the center of the conductor it is given by:

$$
B=i \frac{\mu_{0}}{2 \pi r}
$$

The magnetic induction for a closed path in the magnetic field can be integrated at the surface $S$, resulting in the total flux $\Phi$ :

$$
\Phi=\oint \vec{B} \cdot \overrightarrow{d S}
$$

Here, $\Phi$ is the flux in $\mathrm{Wb}$ and $d S$ is the incremental surface area.

The Ampere's Law allows to calculate the strength of the magnetic field in the form:

$$
\begin{gathered}
\oint \vec{B} \cdot \overrightarrow{d S}=\mu_{0} \cdot I_{\text {enclosed }} \\
\text { B. } L=\mu_{0} . \text { I.n. } L \Rightarrow B=\mu_{0} I n
\end{gathered}
$$

Here $I$ is the current through the wire and $n$ is the number of turns per meters.

A coil of radius $\mathrm{R}$, carries a current $I$. It creates an incremental magnetic field due to each tiny segment of the coil, given by:

$$
\overrightarrow{d B}=\frac{I \cdot \mu_{0}}{4 \pi} \frac{\overrightarrow{d S .} \hat{r}}{r^{2}}
$$

When the vertical components are canceled, then:

$$
d B_{\text {Horizontal }}=\frac{I \mu_{0}}{4 \pi} \frac{d S}{D^{2}+R^{2}} \frac{R}{\left(D^{2}+R^{2}\right)^{1 / 2}}
$$

Here $\mathrm{D}$ is the distance between a generic point $\mathrm{P}$ at the coil axis and the top edge of the coil, and $\mathrm{R}$ is the coil radius. Integrating over every segment to find the total magnetic field, gives:

$$
\vec{B}=\frac{I \mu_{0}}{4 \pi} \frac{R}{\left(D^{2}+R^{2}\right)^{3 / 2}} \int d S
$$

The mutual inductance $M$ as function of the coupling coefficient $k_{T R}$ is expressed by the following equation:

$$
M=k_{T R}\left(L_{T} L_{R}\right)^{1 / 2}
$$

Here, $L_{T}$ is the transmitter coil inductance, and $L_{R}$ is the receiver coil inductance, which are mutually coupled.

The coupling coefficient is a non-dimensional quantity having values between 0 and 1 . In WPT systems the coupling coefficient values are typically very small, close to zero due to the weak magnetic flux connection.

\section{EXPERIMENTAL PART}

The study presented in this paper was performed in the Laboratory of Power Electronics, Faculty of Science and Technology, University Nova of Lisbon, Portugal. It shows the behavior of the three-phase magnetic field measured on a prototype (a scaled physical model) of the WPT system. The experiments were conducted separately in star and delta electrical connections at a frequency of $50 \mathrm{~Hz}$. For the prototype, the geometric configuration of the primary has three coils and has been placed in two different configurations: star and delta. The magnetic field data were measured both in star and delta electrical connection and in both geometric configurations, star and delta, respectively. The three primary coils, with parallelepiped shape, were manufactured by winding 110 turns in two layers, the first layer with 60 turns and the second layer with 50 turns. The length of each core is $180 \mathrm{~mm}$, its height is $300 \mathrm{~mm}$ and the thickness is $60 \mathrm{~mm}$. The primary coils are identified by colors: red, yellow and white as 
shown in Fig. 2 and Fig. 3. The secondary coil (probe coil) consists of ferromagnetic laminated core material, cylindrical shape, with a length of $130 \mathrm{~mm}$ and a diameter of $28 \mathrm{~mm}$. The coil probe was manufactured by winding 180 turns: 4 layers of 40 turns each and the last of 20 turns. It has inductance of $3.56 \mathrm{mH}$ and resistance of $0.7 \Omega$. To conduct the tests a platform was built with a non-ferromagnetic material, namely Kline material, with the following dimensions $1000 \mathrm{~mm}$ of length and $700 \mathrm{~mm}$ of width. The platform is shown in Fig. 1.

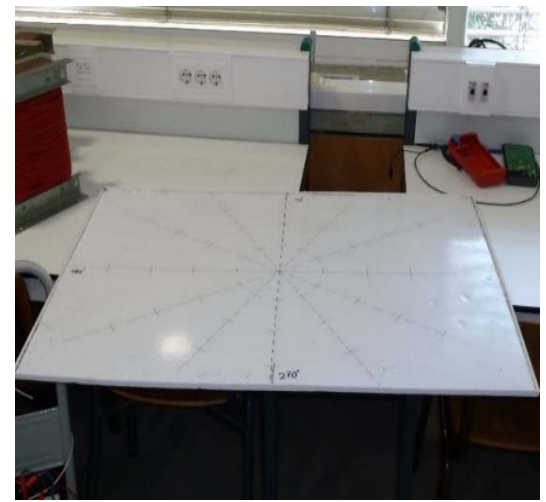

Fig. 1. The platform built in Kline material.

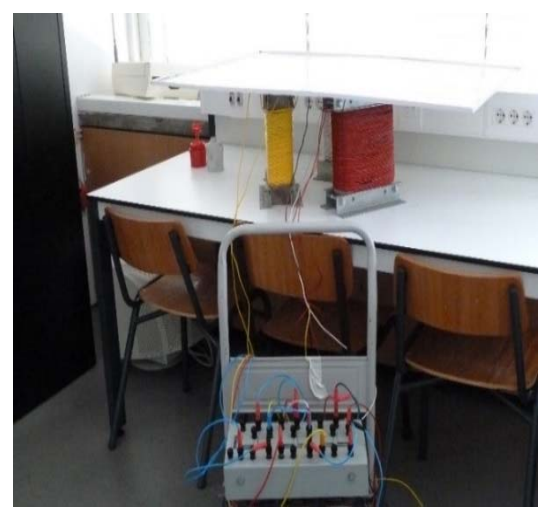

Fig. 2. Prototype built for the primary coils in delta geometric configuration.

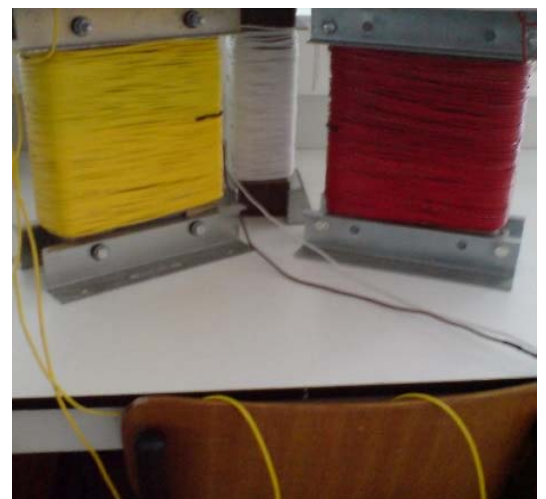

Fig. 3. The primary coils in star geometric configuration.

Over the platform surface, two sets of lines were designed, the first set is made of centered lines at right angle, and the second set consists of radial lines at successive angles of $30^{\circ}$. The radial distances are marked at $100 \mathrm{~mm}, 200 \mathrm{~mm}$ and
$300 \mathrm{~mm}$. With the prototype was performed a significant set of tests that enables the mapping of the magnetic field, in $\mathrm{X}, \mathrm{Y}$ and $\mathrm{Z}$ coordinates, over a circular surface of $28,27 \mathrm{dm}^{2}$. For the tests, the measured peak to peak values at each radial distance were obtained in steps of $30^{\circ}$. The prototype is shown in Figs. 2, 3 and 4. The two geometric configurations, delta and star are shown in figures 2 and 3.

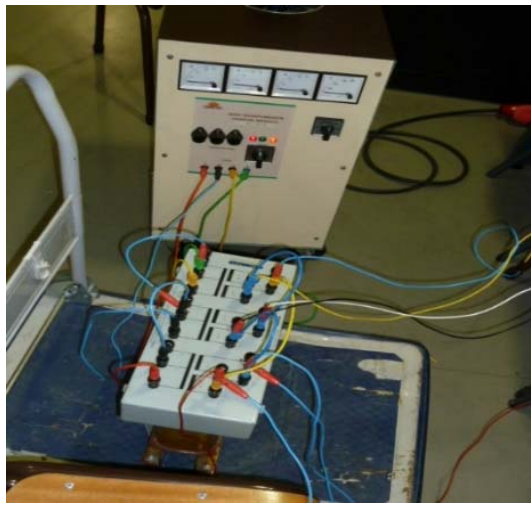

Fig. 4. Three-phase variable transformer and isolated transformer.

Table I summarizes the data measured for the primary windings.

Table II summarizes the data for the inductive reactance and the impedance calculated for the primary windings.

TABLE I.

DATA MEASURED FOR THE PRIMARY WINDINGS

\begin{tabular}{|l|c|c|}
\hline Coil & $\begin{array}{c}\text { Inductan } \\
\text { ce } \\
{[\mathbf{m H}]}\end{array}$ & $\begin{array}{c}\text { Resistanc } \\
\mathbf{e} \\
{[\Omega]}\end{array}$ \\
\hline Red & 8.6 & 1.7 \\
\hline Yellow & 8.66 & 1.6 \\
\hline White & 9.43 & 1.6 \\
\hline
\end{tabular}

TABLE II.

PRIMARY, INDUCTIVE REACTANCE AND IMPEDANCE

\begin{tabular}{|l|c|c|}
\hline Coil & $\begin{array}{c}\text { Inductive } \\
\text { reactance } \\
{[\Omega]}\end{array}$ & $\begin{array}{c}\text { Impedanc } \\
\mathbf{e} \\
{[\Omega]}\end{array}$ \\
\hline Red & 2.7 & 3.19 \\
\hline Yellow & 2.72 & 3.16 \\
\hline White & 2.96 & 3.37 \\
\hline
\end{tabular}

The tests were conducted for the primary input voltages of $20 \mathrm{~V}$ and of $30 \mathrm{~V}$ in delta and star electrical configurations. The geometric configurations of the primary coils are placed in delta or star, respectively. The probe coil is placed in three positions: vertical (figure 5), horizontal longitudinal (figure 6) and horizontal transversal (figure 7) in respect to radial lines.

Table III and Table IV summarize the currents in each primary coil for the input voltages of $20 \mathrm{~V}$ and of $30 \mathrm{~V}$ applied by a variable transformer in star and delta electrical configurations. 
TABLE III.

CURRENTS IN EACH Primary COIL IN STAR EleCtrical CONFIGURATION

\begin{tabular}{|l|c|c|}
\hline Coil & $\begin{array}{c}\text { 20 V } \\
\text { Current } \\
{[\mathbf{A}]}\end{array}$ & $\begin{array}{c}\text { Current } \\
{[\mathbf{A}]}\end{array}$ \\
\hline Red & 6.3 & 9.4 \\
\hline Yellow & 6.3 & 9.5 \\
\hline White & 5.9 & 8.9 \\
\hline
\end{tabular}

TABLE IV.

CURRENTS IN EACH PRIMARY COIL IN DELTA ELECTRICAL CONFIGURATION

\begin{tabular}{|c|c|c|}
\hline Coil & $\begin{array}{c}\text { 20 V } \\
\text { Current } \\
{[\mathbf{A}]}\end{array}$ & $\begin{array}{c}\text { Current } \\
{[\mathbf{A}]}\end{array}$ \\
\hline Red & 10.8 & 16.3 \\
\hline Yellow & 10.9 & 16.5 \\
\hline White & 10.3 & 15.4 \\
\hline
\end{tabular}

\section{CASE STUDY}

The experiments to collect the data were executed with two input voltages, 20Volt and 30Volt applied by a variable transformer as shown in Fig. 4. At each test point the values were obtained with the probe in three positions, vertical, horizontal longitudinal and horizontal transversal as shown in Fig. 5, Fig. 6 and Fig. 7, around the three circumferences with radius $100 \mathrm{~mm}, 200 \mathrm{~mm}$ and $300 \mathrm{~mm}$. The blue, orange and grey lines correspond respectively to the radii $(100 \mathrm{~mm}, 200 \mathrm{~mm}$ and $300 \mathrm{~mm}$ ) at which the rms voltage is measured.

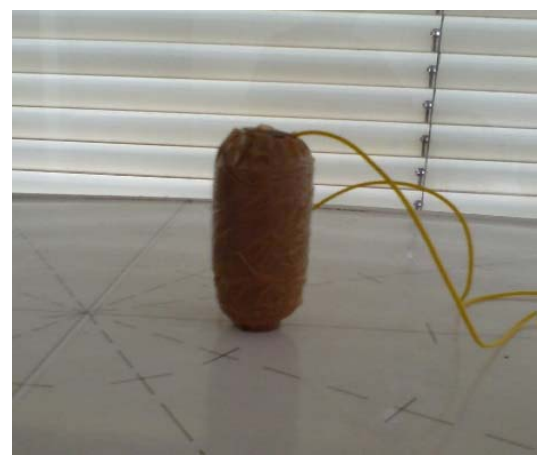

Fig. 5. The probe in vertical position.

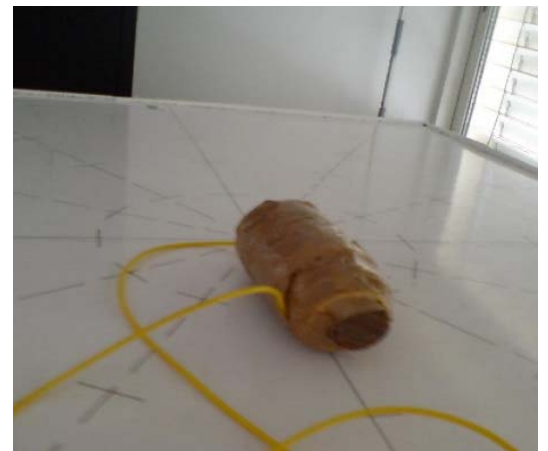

Fig. 6. The probe in horizontal longitudinal position.

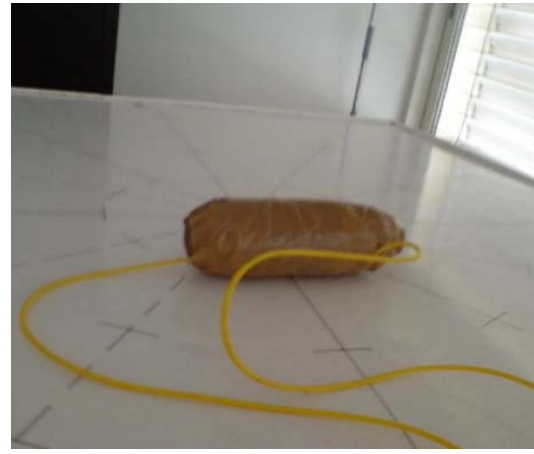

Fig. 7. The probe in horizontal transversal position.

The Figs. 8 and 9 show the voltages collected in same electrical configuration but in two different geometric configurations, first in star and the second in delta. In both tests the probe was placed in vertical position.

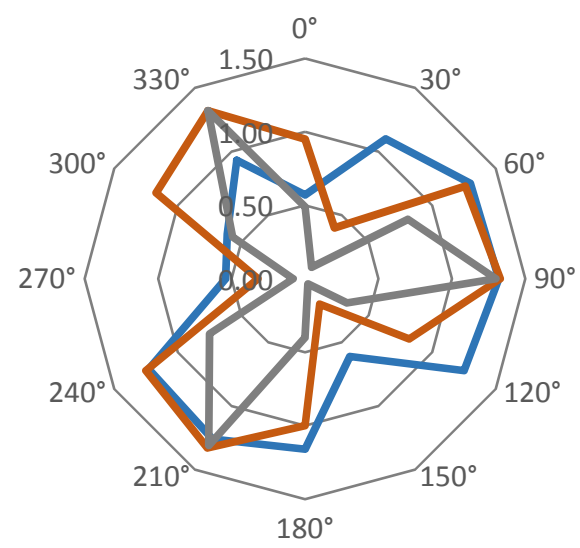

Fig. 8. Star electrical and geometric configurations, probe in vertical position, $\mathrm{Z}=0.0 \mathrm{~cm}$ and input $30 \mathrm{Volt}$

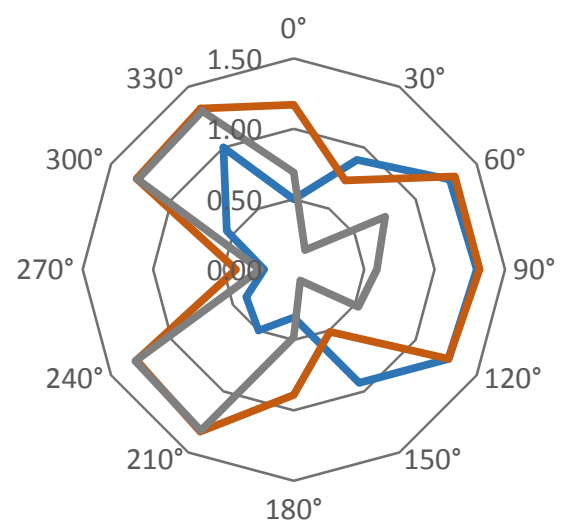

Fig. 9. Star electrical configuration, delta geometric configuration, probe in vertical position, $Z=0,0 \mathrm{~cm}$ and input $30 \mathrm{Volt}$

Comparing the curves of Fig. 10 and Fig. 11 with the curves of Fig. 12 and Fig. 13, it is found that the voltage values obtained from the probe are significantly higher in electric delta configuration. The voltage values in geometric star configuration are always lower when compared to the 
geometric delta configuration regardless of their electrical star or delta configuration. This can be confirmed by Figs. 12 and 13 .

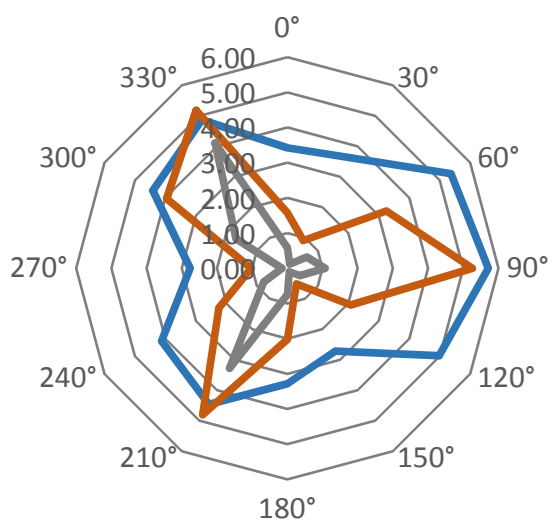

Fig. 10. Delta electrical configuration, star geometric configuration, probe in vertical position, $Z=0,0 \mathrm{~cm}$ and input $30 \mathrm{~V}$

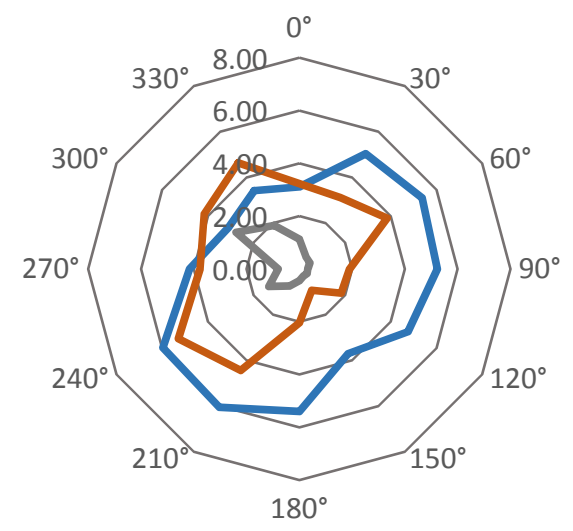

Fig. 11. Delta electrical and geometric configurations, probe in vertical position, $\mathrm{Z}=0,0 \mathrm{~cm}$ and input $30 \mathrm{Volt}$

The voltage values obtained by the probe at a vertical distance $Z=2.5 \mathrm{~cm}$ from the primary coils are shown in Figs. 14 and 15 for the delta electrical and geometric configuration. However, the Fig. 14 shows the voltage values with the probe in vertical position, while in the Fig. 15 the values were obtained with the probe in the horizontal longitudinal position. The voltage values obtained by the probe in vertical position remain higher as were the curves in the previous, Figs. 12 and 13. It is noticeable, that the induced voltage values obtained at a large radial distance (larger circumference), are higher if the probe is in a horizontal longitudinal position, compared to the results of the probe in vertical position (grey line). This shows that at a shorter distance from the transmitter inductors, the lines are mostly vertical, and at a larger distance the lines are mostly horizontal.

The voltage values shown in Figs. 16 and 17, are obtained in the same configuration as applied to the Figs.14 and 15, but at a vertical distance from the transmitter of $Z=5.0 \mathrm{~cm}$. As it is expected, when the vertical distance from the primary coils $Z$ is increased, the voltage values collected by the probe decrease. This can be seen by comparing the curves presented in Fig. 14 and 15. It is noted that at the distance $Z=5.0 \mathrm{~cm}$ the curves become more circular, showing a magnetic field more equally distributed. As in the previous Figs. 14 and 15 (a probe in horizontal longitudinal position), the collected voltage values at an outer circumference (grey line) are higher (in Fig. 16, 17).

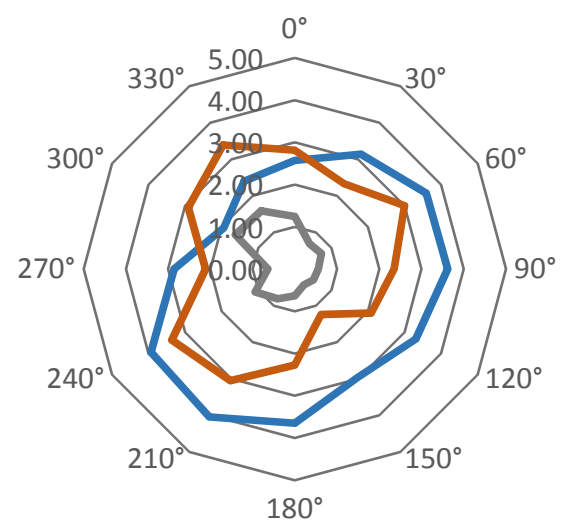

Fig. 12. Delta electrical and geometric configurations, probe in vertical position, $\mathrm{Z}=2.5 \mathrm{~cm}$ and input $30 \mathrm{Volt}$

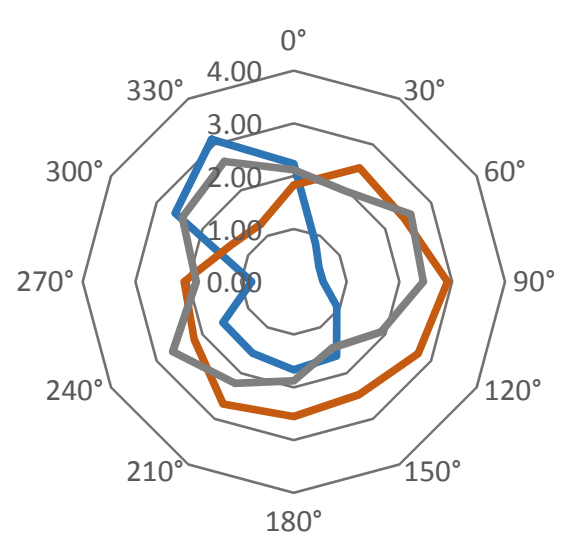

Fig. 13. Delta electrical and geometric configurations, probe in horizontal longitudinal position, $\mathrm{Z}=2.5 \mathrm{~cm}$ and input $30 \mathrm{Volt}$

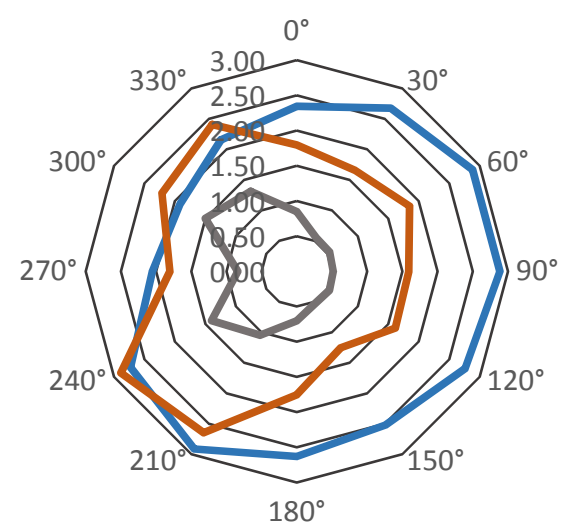

Fig. 14. Delta electrical and geometric configurations, probe in vertical position, $\mathrm{Z}=5.0 \mathrm{~cm}$ and input $30 \mathrm{Volt}$ 


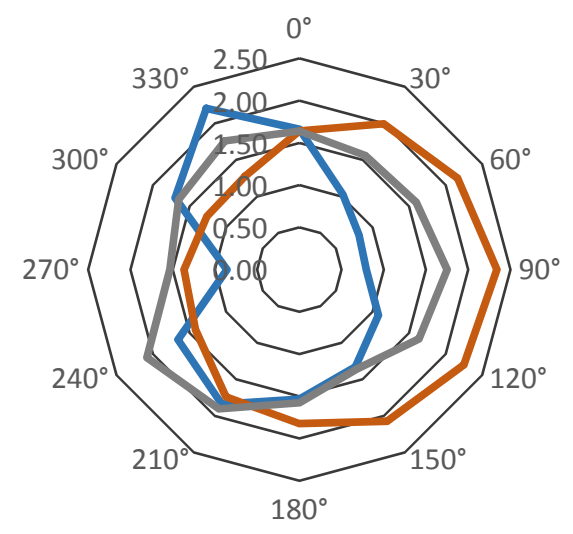

Fig. 15. Delta electrical and geometric configurations, probe in horizontal longitudinal position, $\mathrm{Z}=5.0 \mathrm{~cm}$ and input $30 \mathrm{Volt}$

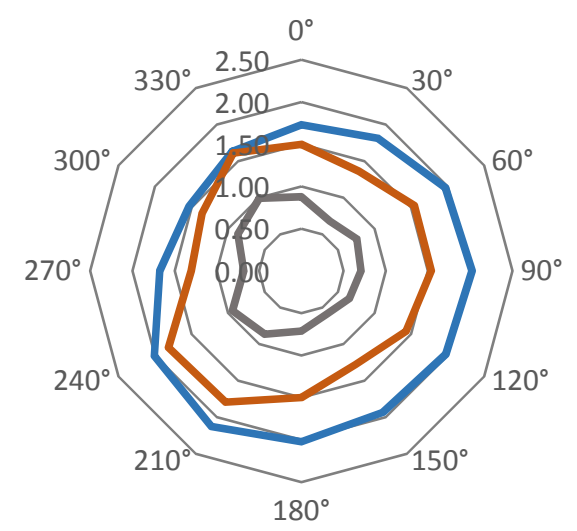

Fig. 16. Delta electrical and geometric configurations, probe in vertical position, $\mathrm{Z}=10.0 \mathrm{~cm}$ and input $30 \mathrm{Volt}$

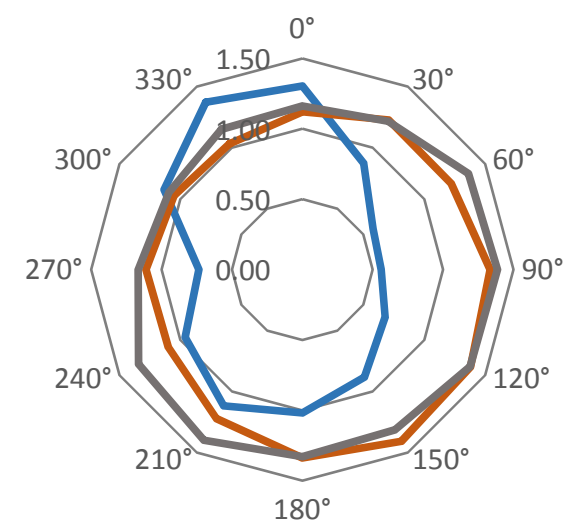

Fig. 17. Delta electrical and geometric configurations, probe in horizontal longitudinal position, $\mathrm{Z}=10.0 \mathrm{~cm}$ and input $30 \mathrm{Volt}$

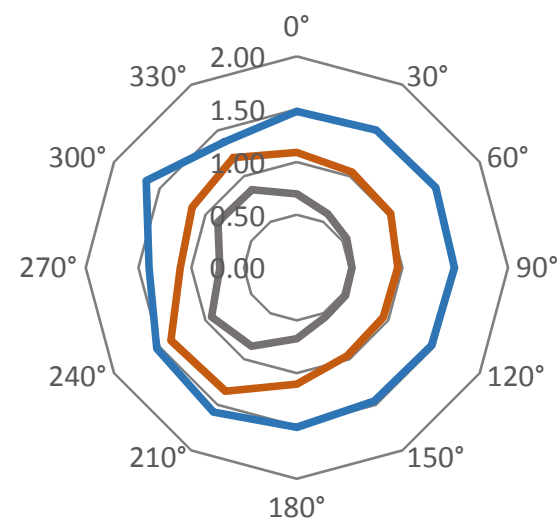

Fig. 18. Delta electrical and geometric configurations, probe in vertical position, $\mathrm{Z}=15.0 \mathrm{~cm}$ and input $30 \mathrm{Volt}$

The Figs. 18 and 19 show the voltage values obtained by the probe at a vertical distance $Z=10.0 \mathrm{~cm}$ from the primary coils. The electric and geometric configuration was maintained in delta. In Fig. 18, the voltage values obtained by the probe in vertical position are shown, while in Fig. 19 the voltage values are shown from the probe in horizontal longitudinal position

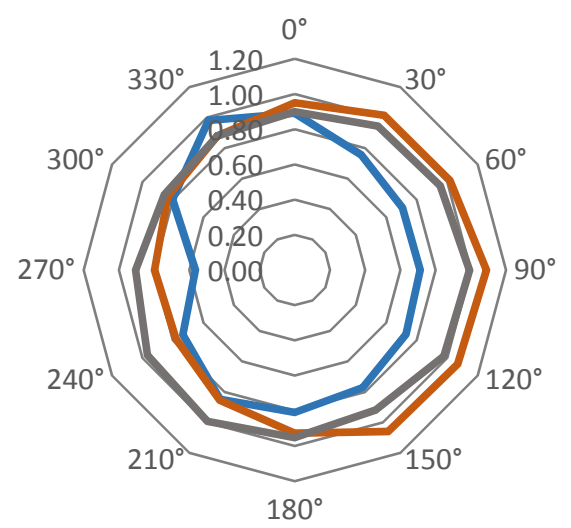

Fig. 19. Delta electrical and geometric configurations, probe in horizontal longitudinal position, $Z=15.0 \mathrm{~cm}$ and input 30 Volt

The curves, obtained by the probe at a vertical distance $Z=15.0 \mathrm{~cm}$ from the primary coils, were left out of this report, as the voltage values were lower and not showing something important and new. The test conditions for the $Z=15.0 \mathrm{~cm}$ were exactly the same as in the previously reported figures and it is verified that all the observations made for Fig.18 and 19, are also valid at this $15.0 \mathrm{~cm}$ distance.

\section{CONCLUSIONS}

In conclusion, the delta electrical and geometric configuration is the best solution for the transmitter of the WPT in three-phase systems. In order to optimize the transfer system, the receptor coils should be in vertical position. The term "coil" is mentioned here because the axis orientation of the receiving inductor is to be considered, and this is not 
depending on the existence of a ferromagnetic concentrator (a magnetic core).

At shorter distances between the transmitter and the receiver coils (e.g. $Z=2.5 \mathrm{~cm}$ ), higher induced voltage values are obtained, and a hence, a higher magnetic intensity of the magnetic field is observed, but the alignment problems are more critical (the field is more concentrated near to the transmitting coils). From the measurements it is found that to achieve significant voltage values, a circle of radius $200 \mathrm{~mm}$ is a limit. For radial distance greater than this value, the voltage values are lower, as it should be expected, but the horizontally placed receiving coil shows better results at the larger radial distance. Maintaining the same electrical and geometric configuration, but with the receiving coil in the horizontal longitudinal position, the voltage values are relatively lower when compared to the coils in vertical position. However, at a larger radial distance the horizontally positioned receiver has a comparable value of induced voltage. What is more important, the alignment problems are less critical, the magnetic field is more uniform, and uniform induced voltage is obtained at a circle of radius $300 \mathrm{~mm}$ (diameter of $0.6 \mathrm{~m}$ ), even for vertical distances up to $15 \mathrm{~cm}$.

The measurements in this physical model were made at frequency of $50 \mathrm{~Hz}$. Considering the behavior of the magnetic parameters at higher frequencies, the results in a higher frequency system will be way better.

\section{REFERENCIES}

[1] A. Karalis, J.D Joannopoulos, M. Soljačic, "Efficient Wireless nonradiative Mid-Range Energy Transfer", Annals of Physics 323 (2008) pp. $34-48$

[2] R. Bhutkar, S. Sapre, "Wireless Energy Transfer Using Magnetic Resonance" 2009 Second International Conference on Computer and electrical Engineering, IEEE computer Society

[3] J. Wu, Y. Bingnan, T. William, H. Koon, "Circularly Polarized Near Field for Resonant Wireless Power Transfer" Publiched in: IEEE Wireless Power Transfer Conference (WPTC) 2015

[4] T.C. Beh, M. Kato, T. Imura, Y. Hori, "Wireless Power Transfer System Resonant Coupling at Fixed Resonant Frequency - Power Transfer System Based on Impedance Matching". World Electronic Journal Vol 4 - ISSN 2032-6653. EVS 25, World Battery Hybrid and Fuel Cell Electric Vehicle Symposium, November 2010.

[5] S.Y.R. Hui, W.X. Zhong, C.K. Lee, "A Critical Review of Recent Progress in Mid-range wireless Power Transfer". IEEE Transactions on Power electronics, Vol. 29, N. 9. September 2014.

[6] B. Wang, W. Yerazunis, K.H. Teo, "Wireless Power Transfer: Metamaterials and Array of Coupled Resonators". Proceeding of the IEEE, Vol. 101, Issue 6, Pags 1359-1368, ISSN: 0018-9219, June 2013.

[7] A.J. Elliot, G. Covic, S. Raabe, J.T. Boys, "Multiphase Pickups for large Lateral Tolerance Contactless Power-Transfer systems". IEEE transactions on Industrial Electronics, Vol. 57, No. 5, May 2010.

[8] A. Moradewicz, M.P. Kazamierkowski, "High Efficiency Contactless Energy Transfer System with Power Electronic Resonant Converter". Bulletin of the Polish Academy of Sciences - Technical Sciences - Vol. 57, No. 4, 2009

[9] S. Valtchev, B. Borges, K. Brandisky, J.B. Klaassens, "Resonant Contactless Energy Transfer with Improved Efficiency". IEEE Transactions on Power Electronics, Vol. 24, No. 3, March 2009

[10] T. Imura, H. Okabe, Y. Hori, "Basic Experimental Study on Helical Antennas of Wireless Power Transfer for Electric Vehicle by Using Magnetic Resonant Coupling". Published in: Vehicle Power and Propulsion Conference, VPPC '09 IEEE. Pags 936-940. IEEE 2009

[11] X.Z. Wei, Z. Wang, H. Dai, "A critical Review of Wireless Power Transfer via Strong Coupled Magnetic Resonances". Energies 2014, 7, 4316-4341. Published in 7 July 2014.

[12] D.M. Vilathgamuwa, J.P.K. Sampath, "Chapter 2 - Wireless Power Transfer (WPT) for Electric Vehicle (EV's) - Present and Future
Trends". Springer Science+ Business Media Singapore 2015, S. Rajakaruna et al. (eds), Plug In Electric Vehicles in Smart Grids, Power Systems. DOI 10.1007/978-981-287-299-9 2

[13] C. Yang, K. Cheng, K. Tsunekawa, "A Novel Parallel Double Helix Loop Resonator for Magnetic Coupled Resonance Wireless Power Transfer". Progress in Electromagnetics Research Symposium Proceedings, Guangzhou, China, Aug. 25-28, 2014.

[14] T. Imura, Y. Hori, "Maximizing Air Gap and Efficiency of Magnetic Resonant Coupling for Wireless Power transfer Using Equivalent Circuit and Neumann Formula". IEEE Transactions on Industrial Electronics, Vol. 58, No. 10, October 2011.

[15] R.Duarte, F. Mendes, G. Klaric, "Analysis of the Coupling Coefficient in Inductive Energy Transfer Systems". Hindawi Publishing Corporation, Active and Passive Electronic Components, Vol. 2014, Article ID 951624 